\title{
Rinosinusitis maxilar crónica de origen dental
}

\author{
Chronic maxillary rhinosinusitis of dental origin
}

\author{
Claudia Heider C' , Gloria Ribalta L², Daniel Bacigalupe $\mathbf{R}^{3}$, Karin Krauss $\mathbf{M}^{4}$.
}

\begin{abstract}
RESUMEN
Introducción: La rinosinusitis maxilar de origen dental (RSMD) es una entidad reconocida con una incidencia cercana al 30\%. Su diagnóstico puede pasar inadvertido, tanto en la clínica como en las imágenes, llevando a persistencia de sintomatología y fracaso de tratamiento.

Objetivo: Describir la presentación clínica, diagnóstico y tratamiento de pacientes con rinosinusitis maxilar de origen dental.

Material y método: Reporte de casos. Se revisaron historias clínicas, imágenes radiológicas, protocolos operatorios y cultivos microbiológicos de pacientes con diagnóstico de rinosinusitis de origen dental atendidos en Clínica Las Condes.

Resultados: Diez pacientes, siete mujeres y tres hombres con edad promedio de 63 años. Descarga posterior y rinorrea fueron los síntomas más frecuentes. El estudio incluyó radiografía dental y tomografía computarizada de cavidades paranasales. Las complicaciones secundarias a extracciones dentales y de implantes dentales fueron las etiologías más frecuentes, siendo el primer molar la pieza dental más comprometida. Todos los casos fueron manejados en conjunto por equipo médico y odontológico. Ocho pacientes requirieron tratamiento quirúrgico y 5 de ellos además procedimientos dentales asociados. La flora responsable fue mixta aerobia y anaerobia.

Conclusión: En pacientes rebeldes a tratamiento y con síntomas unilaterales, se debe tener un alto índice de sospecha y buscar dirigidamente patologías dentales que expliquen cuadros rinosinusales máxilo-etmoidales.
\end{abstract}

Palabras clave: Sinusitis maxilar, sinusitis odontógena, infección dental.

\begin{abstract}
Introduction: Odontogenic maxillary sinusitis is a well-recognized condition and accounts for approximately $30 \%$ of maxillary sinusitis cases. The diagnosis can be missed leading to failure in medical and surgical treatments.
\end{abstract}

\footnotetext{
Médico Cirujano. Programa de Magíster en Epidemiología Clínica, Universidad de La Frontera.

Médico Otorrinolaringólogo, Clínica Las Condes.

Odontólogo, Clínica Las Condes.

Médico Cirujano.
} 
Aim: To describe clinical features, diagnosis procedures and treatment of odontogenic maxillary sinusitis.

Material and method: Case Report. We retrospectively reviewed medical records, radiologic characteristic, surgical findings and intraoperative microbial cultures of patients with diagnosis of odontogenic sinusitis at Clínica Las Condes.

Results: 10 patients were identified, 7 females and 3 males with a mean age of 63 years. Posterior discharge and rhinorrhea were the most common symptoms. Dental $x$-rays and paranasal sinus CT-scans were performed in all patients. latrogenic causes, which include dental implants and dental extractions, were the most common etiology and the 1st molar was the dental piece most frequently involved. Both otolaryngologists and dentistsworked together in all cases. 8 patients had endoscopic sinus surgery and five of them received concomitant management of the dental origin. Polymicrobial cultures were obtained.

Conclusions: Otolaryngologists must have a high index of suspicion and look for an odontogenic cause in individuals with unilateral maxillary and ethmoidal symptoms, resistant to conventional sinusitis therapy.

Key words: Maxillary Sinusitis, odontogenic sinusitis, dental infections.

\section{INTRODUCCIÓN}

La rinosinusitis de origen dental da cuenta del $10 \%$ al $12 \%$ de los casos de rinosinusitis maxilar ${ }^{1}$ alcanzando en series más actuales hasta el $25 \%{ }^{2} \mathrm{e}$ incluso $40 \%{ }^{3}$. Su incidencia exacta es desconocida y difícil de determinar pero existe evidencia que demuestra que se encuentra en aumento ${ }^{4}$.

Debido a su ubicación anatómica, el seno maxilar se encuentra vulnerable a la invasión de microorganismos patógenos tanto de la cavidad oral como nasal. Si bien dependerá del tamaño del seno y de su grado de neumatización, existe una estrecha relación entre las raíces del segundo y primer molar superior, seguido por los premolares, con el piso del seno maxilar. Es importante destacar que estas raíces se encuentran separadas del seno por hueso cortical de espesor variable, (alrededor de $2 \mathrm{~mm})^{5}$ y que en ocasiones, éstas pueden protruir a la cavidad sinusal quedando sólo cubiertas por el mucoperiostio del seno (membrana de Schneiderian) ${ }^{6}$. Existen además numerosas anastomosis vasculares que perforan este espacio y pueden servir de canales para la propagación de bacterias, facilitando la extensión de la infección hacia el seno maxilar?.

La mayoría de las infecciones sinusales asociadas a causa odontológica resultan de la perforación de esta membrana secundaria a procesos infecciosos originados a partir de los dientes superiores adyacentes al seno maxilar (caries, pulpitis, absceso periapical, enfermedad periodontal). Otras patologías pueden ser el trauma dental, la patología odontológica del hueso maxilar y las causas iatrogénicas como extracciones dentales, osteotomías maxilares en cirugía ortognática y colocación de implantes dentales 8 .

Según un metaanálisis publicado en relación a la etiología de la sinusitis maxilar odontógena (Tabla 1) la causa más frecuente es la iatrogenia $(55,97 \%)$, siendo las complicaciones postexodoncia (fístula oro antral, resto radicular) responsables del $47 \%$ de ellas 9 .

A pesar de ser una causa reconocida de sinusitis maxilar, existen pocas referencias y recomendaciones para su diagnóstico y tratamiento. Según una búsqueda bibliográfica llevada a cabo en 2010 , sólo el $13 \%$ (11/85) de las guías de práctica clínica publicadas hasta esa fecha mencionan la patología dental como causa de rinosinusitis crónica y tres recomiendan procedimientos diagnósticos ${ }^{10}$.

Debido a que tanto su fisiopatología, microbiología y manejo difieren de las infecciones sinusales habituales, es importante reconocerla y tratarla oportunamente. Su diagnóstico puede pasar inicialmente inadvertido, tanto en la clínica como en las imágenes, llevando a persistencia de sintomatología, 
Tabla 1. Etiología rinosinusitis de origen dental

\begin{tabular}{|lr|}
\hline Causa & Porcentaje (\%) \\
\hline latrogenia & 55,97 \\
Posextracción dental & 47,56 \\
Reparación fístula oro-antral, cuerpo extraño inespecífico & 19,72 \\
Extrusión de material de obturación en endodoncia hacia el seno maxilar & 22,27 \\
Restos de amalgama posapicectomía & 5,33 \\
Elevación seno maxilar en cirugía preimplante & 4,17 \\
Complicaciones de implantes dentales (migración, mal posición) & 0,92 \\
Periodontitis & 40,38 \\
Quiste odontogénico & 6,66 \\
\hline
\end{tabular}

Adaptado de Arias 0, Barona C et al. Meta-analysis of the etiology of odontogenic maxillary sinusitis. Med Oral Patol Oral Cir Bucal 2010; 15(1): e70-3.

mala respuesta a tratamiento antibiótico e incluso a fracaso de la cirugía endoscópica nasal realizada.

\section{OBJETIVO}

El objetivo de este trabajo es describir la presentación clínica, el diagnóstico y tratamiento de una serie de pacientes atendidos en Clínica Las Condes con diagnóstico de rinosinusitis maxilar de origen dental, así como alertar a los especialistas de otorrinolaringología, radiología y odontología, sobre la alta incidencia de diagnóstico errado en esta patología que lleva a los pacientes a deambular entre varios especialistas hasta llegar al diagnóstico correcto y tratamiento definitivo.

\section{MATERIAL Y MÉTODO}

Se presentan 10 casos atendidos en el Departamento de Otorrinolaringología de Clínica Las Condes entre los años 2000 y 2013 con diagnóstico de rinosinusitis maxilar crónica de origen dental (RSMD). El diagnóstico se realizó en base a la evaluación médica y odontológica, que incluyó la evaluación de los síntomas (según los criterios diagnósticos planteados por la Academia Americana de Otorrinolaringología y Cirugía de Cabeza y Cuello (AA0-HNS), la historia dental previa y los hallazgos radiológicos de la radiografía dental y la tomografía de cavidades paranasales. Además, se contó con la colaboración de odontólogos del mismo centro, quienes apoyaron en el proceso diagnóstico.
Se registraron características biodemográficas, presentación clínica de la enfermedad, factores etiológicos, hallazgos radiológicos, tratamiento realizado y evolución clínica. Se utilizó estadística descriptiva. (Medidas de tendencia central y rangos).

\section{RESULTADOS}

Se estudiaron un total de 10 pacientes: Siete mujeres y tres hombres con una edad promedio de 63 años $\pm 9,8$ (48 a 84 años). El tiempo de evolución de los síntomas presentó una mediana de 17,5 meses $(0,5 \mathrm{a}$ 192). Los síntomas más frecuentes fueron: descarga posterior y rinorrea fétida (Tabla 2). Sólo un paciente presentó dolor dental.

Los pacientes fueron estudiados con radiografía dental y tomografía computarizada de cavidades paranasales (TC CPN). Los informes radiológicos dentales no fueron concluyentes, mientras que en la TC CPN, la patología odon-

Tabla 2. Presentación clínica

\begin{tabular}{|lcc|}
\hline & $\mathrm{n}$ & $\%$ \\
\hline Descarga posterior & $7 / 10$ & 70 \\
Rinorrea & $5 / 10$ & 50 \\
Halitosis & $4 / 10$ & 40 \\
Cefalea fronto orbitaria & $4 / 10$ & 40 \\
Dolor maxilar & $3 / 10$ & 30 \\
Obstrucción nasal & $2 / 10$ & 20 \\
Dolor dental & $1 / 10$ & 10 \\
\hline
\end{tabular}


Tabla 3. Etiología

\begin{tabular}{|ccllr|}
\hline Caso & Edad & Etiología dental & Tratamiento Odontológico & Pieza \\
\hline 1 & 63 & Fístula oroantral & Cierre de fístula con desplazamiento de colgajo vestibular. & 3 \\
2 & 59 & Caries & Extracción pieza & 15 \\
3 & 69 & $\begin{array}{l}\text { Complicación implante. } \\
\text { Fístula oroantral. }\end{array}$ & $\begin{array}{l}\text { Remoción tornillos implantes. } \\
\text { Cierre de fístula con colgajo. }\end{array}$ \\
& & Implante intrasinusal & Se conserva implante al descartar presencia de fístula & 3 \\
4 & 84 & Quiste dentígeno. & Paciente rechaza cirugía. & $13-14$ \\
5 & 70 & Fístula oroantral & Observación & 14 \\
6 & 62 & Osté́tis periapical/ & retratamiento endodóntico & 15 \\
7 & 48 & Caries & Apicectomía pieza & 14 \\
8 & 64 & Implante intrasinusal & Se conserva implante al descartar presencia de fístula & $3-4$ \\
9 & 54 & Fistula oroantral & Se descarta presencia de fístula & $12-13$ \\
10 & 58 & Resto radicular & Extracción resto radicular & 14 \\
\hline
\end{tabular}

tológica asociada al seno maxilar fue evidente en el $50 \%$ de los casos, pero sólo en uno de ellos informada por el radiólogo. El hallazgo más frecuente fue el velamiento completo del seno maxilar ipsilateral a la enfermedad odontológica asociada, siendo en nuestra serie el seno maxilar izquierdo el más frecuentemente comprometido $(70 \%)$. En todos los casos hubo además ocupamiento parcial de etmoides ipsilateral con obliteración del complejo osteomeatal (COM) por inflamación. Sólo un paciente requirió evaluación adicional con tomografía computarizada dental para llegar al diagnóstico.

Con respecto a la etiología dental (Tabla 3), las complicaciones secundarias a extracciones dentales (restos radiculares, fístulas oroantrales) $(5 / 10)$ y de los implantes dentales (3/10) fueron las más frecuentes. Otras causas como complicaciones secundarias a caries y quiste dentígeno fueron encontradas con menor frecuencia. Las piezas más comprometidas fueron: primer molar (7 casos), segundo premolar (3 casos), segundo molar (2 casos) y primer premolar (1 caso).

Con respecto al tratamiento, todos los pacientes fueron evaluados en conjunto por otorrinolaringólogo y odontólogo. Ocho pacientes fueron sometidos a cirugía, un paciente rechazó el tratamiento quirúrgico propuesto, y otro se encuentra en observación. En los ocho casos que requirieron intervención quirúrgica se realizó cirugía endoscópica funcional del seno maxilar comprometido; antrostomía, toma de cultivos y lavados exhaustivos con suero y gentamicina para erradicar los probables biofilms. No se abrió bula etmoidal. Lo anterior se llevó a cabo en conjunto con la revisión dental intraoperatoria por el odontólogo tratante. Cinco de ellos requirieron concomitantemente procedimientos odontológicos para el tratamiento de la patología dental asociada ya sea mediante la reparación de fístula a través de avance de colgajos o extracciones dentales de las piezas comprometidas. Durante el seguimiento de estos pacientes no se han detectado recurrencias.

En todos los pacientes sometidos a cirugía se obtuvieron muestras para cultivo aerobio, anaerobio y hongos. Los microorganismos aislados se describen en la Tabla 4.

Tabla 4. Microbiología

\begin{tabular}{|lc|}
\hline Microorganismo & Número de aislados \\
\hline Staphylococcus aureus & 1 \\
Klebsiella pneumoniae & 1 \\
Peptostreptococcus & 2 \\
Pseudomonas aeruginosa & 1 \\
Prevotella spp & 1 \\
Streptococcus viridans & 2 \\
Candida albicans & 2 \\
Streptococcus beta hemolítico grupo F & 1 \\
\hline
\end{tabular}


Con respecto a la histopatología se lograron rescatar seis muestras de biopsias, siendo la inflamación crónica inespecífica el hallazgo encontrado con mayor frecuencia (4/6). En dos casos se encontró en la biopsia micetoma por aspergillus.

\section{DISCUSIÓN}

Según estudios internacionales ${ }^{9}$ la RSMD es una condición diagnosticada con mayor frecuencia en mujeres por sobre los 50 años. En nuestra serie, los resultados no difieren de lo encontrado en la literatura, correspondiendo el $70 \%$ a mujeres con un promedio de edad en la sexta década de la vida.

La presentación clínica tampoco difiere mayormente de los síntomas habituales de rinosinusitis de otras etiologías, siendo el más frecuente en este estudio la descarga posterior. Los síntomas clásicamente descritos como sugerentes de etiología dental (halitosis, cefalea) fueron pesquisados con menor frecuencia. Sólo un paciente refirió dolor dental. Brook ${ }^{11}$ también reportó que el dolor dental es un síntoma infrecuente en RSMD, y por tanto, ni específico ni sensible para el diagnóstico, pudiendo encontrarse también en rinosinusitis no odontogénica ${ }^{10}$. Otras series tampoco han encontrado diferencias en la presentación clinica. Lee ${ }^{12}$ estudió 27 pacientes con diagnóstico de RSMD, siendo el síntoma más habitual la rinorrea puruIenta unilateral $(66,7 \%)$ seguido por el dolor facial $(33,3 \%)$. No encontraron diferencias significativas entre los síntomas de RSMD en comparación con otros tipos de rinosinusitis.

El diagnóstico de RSMD se basa en un acucioso examen otorrinolaringológico y dental que incluye una evaluación de los síntomas del paciente y de su historia dental previa. Dado que la clínica puede resultar inespecífica, es fundamental un alto índice de sospecha y una anamnesis detallada y específicamente dirigida a las intervenciones dentales previas del paciente: preguntar por tratamiento de conducto, implantes dentales, historia de infecciones dentales o cirugías dentoalveolares, entre otras.

Las imágenes radiológicas son una herramienta indispensable para establecer el diag- nóstico. En nuestra serie, todos los pacientes se evaluaron con radiografías dentales y TC CPN. Con respecto a la radiografía dental clásica (panorámica) su utilidad es limitada y hasta el $86 \%$ de los pacientes con RSMD presentan imágenes normales ${ }^{10}$. Esta modalidad tiene una sensibilidad de $60 \%$ para caries y $85 \%$ para enfermedad periodontal ${ }^{13}$ y tiende a subestimar el grado de compromiso óseo. Aunque en nuestros pacientes las placas dentales resultaron no concluyentes, permite evaluar la relación de piezas dentales superiores con el seno maxilar, el grado de neumatización, la presencia de pseudoquistes y la identificación de cuerpos extraños, raíces 0 piezas dentales desplazadas ${ }^{6}$.

La TC CPN resulta el método de elección para visualizar el seno maxilar y entrega mayor información con respecto a la localización anatómica exacta de la patología dental y su relación con el piso del seno maxilar. El hallazgo más frecuente encontrado fue el velamiento completo del seno maxilar ipsilateral a la enfermedad odontológica asociada, situación que se correlaciona con lo encontrado en la literatura ${ }^{10}$. En un análisis de 202 senos maxilares, Bomeli ${ }^{14}$ encontró una relación entre la extensión de la opacificación maxilar y el engrosamiento mucoso con la incidencia de RSMD, demostrando que la probabilidad de encontrar una causa dental subyacente aumenta dramáticamente a medida que aumenta la extensión del velamiento del seno maxilar comprometido. En aquellos senos con una velamiento mayor a dos tercios y engrosamiento mucoso moderado la probabilidad de encontrar una causa dental identificable puede alcanzar el $86 \%$. A pesar de ser una excelente alternativa, se debe considerar que en hasta el $67 \%{ }^{10}$ de los casos, los informes radiológicos iniciales de pacientes con RSMD no dan cuenta de la alteraciones dentales. En una serie de Longuini ${ }^{10}$ de 21 pacientes con RSMD, sólo en el 30\% de los casos la patología dental fue informada inicialmente por el radiólogo, situación similar a la observada en nuestra serie. En este punto los otorrinolaringólogos deben ser cuidadosos y revisar personalmente y con atención las imágenes tomográficas, especialmente de aquellos pacientes con cuadros unilaterales y refractarios a tratamiento habitual, en busca de patologías 
dentales que expliquen el cuadro sinusal y que pueden pasar inadvertidas tanto para odontológos como radiólogos.

Otras alternativas que pueden ayudar a identificar la patología dental son las tomografías computarizadas dentales de alta resolución y el Cone Beam Volumetric $C T^{15-17}$. Este último ha ganado popularidad entre los odontólogos, especialmente en el área de la implantología, debido a que aporta baja dosis de radiación y ofrece imágenes de mejor resolución y en tres dimensiones en comparación con la tomografía computarizada. Su utilidad en el diagnóstico etiológico de RSMD ha sido demostrada en pequeñas series de casos, y su uso podría ayudar a planear adecuadamente tratamientos quirúrgicos complejos. Su alto costo limita sus aplicaciones en la actualidad.

La causa más frecuente de RSMD en nuestra serie fueron complicaciones secundarias a extracciones dentales e implantes dentales. Esta situación también descrita en la literatura ${ }^{9}$, muestra una tendencia hacia un predominio de causas iatrogénicas (aproximadamente 50\%) en contraste con otros factores etiológicos como periodontitis, probablemente en relación con una disminución en la prevalencia de estas patologías debido a la mejoría en la higiene oral de la población. Teniendo en cuenta que las extracciones dentales constituyen una de las actividades realizadas con mayor frecuencia en las clínicas dentales y dada la estrecha relación entre los piezas dentales superiores y el seno maxilar, es razonable encontrar una alta prevalencia de fístulas oroantrales como complicaciones secundarias a extracciones dentales. Especial consideración se debe tener en relación a las complicaciones de los implantes dentales. Su incidencia a pesar de ser aún baja, se encuentra gradualmente en aumento $^{12}$. Lo observado con mayor frecuencia es la infección local de tejido alrededor del implante dental asociado o no a resorción ósea. De esta forma, implantes colocados muy cerca del seno maxilar pueden ofrecer una ruta de infección de la cavidad oral hacia el seno. La migración del implante dental hacia el interior del seno maxilar puede corresponder a otra causa de RSMD, actuando como cuerpo extraño y provocando infección crónica ${ }^{18-20}$ (Figura 1).

Las raíces del segundo molar son las más cercanas al piso del seno maxilar, seguidas en

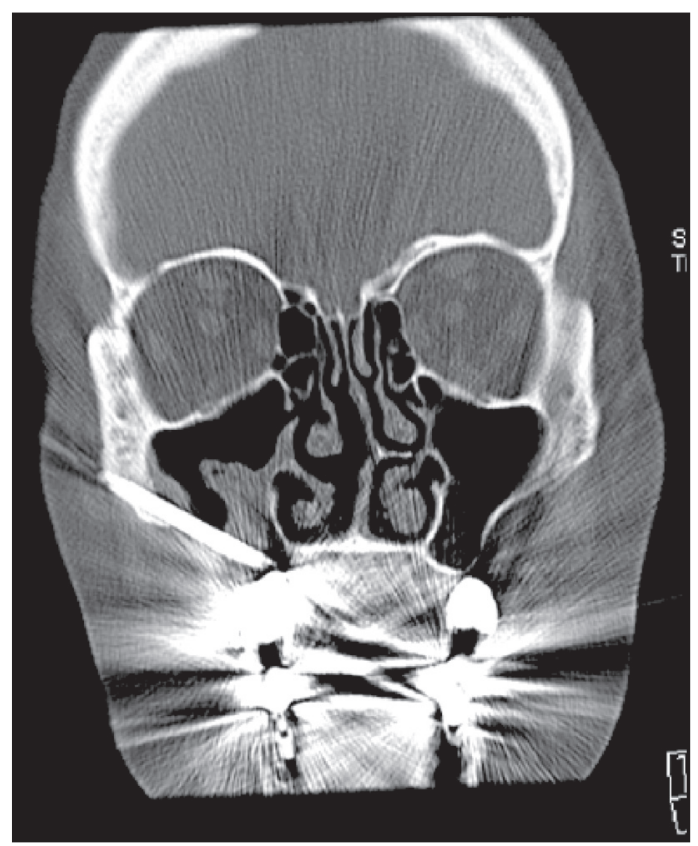

Figura 1. Complicación implante dental. TC CPN que muestra implante dental desplazado que atraviesa el seno maxilar derecho. Se observan cambios inflamatorios ipsilaterales. 
frecuencia por el primer molar, segundo premolar y primer premolar. Esta estrecha relación explica la extensión de procesos infecciosos desde estas piezas dentales hacia el seno maxilar. En nuestra serie, al igual que en el metaanálisis de Arias ${ }^{9}$, el primer molar fue el más comprometido.

Con respecto a los resultados microbiológicos se aprecia una concordancia con los estudios de Brook $^{21}$ y Puglisi22 destacando la presencia de flora polimicrobiana tanto aerobia como anaerobia, siendo en nuestra serie Streptococcus viridans y Peptostreptococcus los microorganismos más frecuentemente aislados. Esto se debe a que la flora oral responsable de las infecciones odontológicas es la misma involucrada en la RSMD y por tanto polimicrobiana. En relación a esto, es importante destacar la importancia de la obtención de muestras de secreciones intraoperatorias del seno maxilar para cultivo, incluyendo técnicas para aerobios, anaerobios y hongos, en tubos adecuados y con rápido transporte al laboratorio de microbiología.

El tratamiento de la RSMD difiere del tratamiento habitual de rinosinusitis de otras etiologías y requiere siempre de una evaluación y tratamiento dental concomitante. Es necesario verificar la permeabilidad del COM en los cortes tomográficos pues ello determinará la conducta quirúrgica. Generalmente se requiere de una combinación de tratamiento médico con cobertura antibiótica adecuada para aerobios y anaerobios, drenaje quirúrgico endoscópico cuando sea indicado y tratamiento odontológico dirigido a la patología causal (ej. remoción de cuerpos extraños, extracción piezas comprometidas) para así evitar la recurrencia. En nuestro estudio, el $80 \%$ de los pacientes se sometió a cirugía endoscópica funcional de senos paranasales con antrostomía del seno maxilar comprometido y revisión dental intraoperatoria, sin necesidad de abrir etmoides. Ante la presencia de fístula oroantral mayor a 5 $\mathrm{mm}$ se requiere además de cirugías orales que permitan el cierre primario de la fístula mediante el avance de colgajos ${ }^{6}$. Se debe destacar la importancia del diagnóstico etiológico, dado que el tratamiento médico antibiótico sin drenaje del seno vía endoscópica y sin eliminación de la fuente de infección por odontólogo, perpetúa el proceso, seleccionando bacterias resistentes y favoreciendo la presencia de hongos. Se debe trabajar en conjunto con el odontólogo para asegurar así la resolución del cuadro y prevenir futuras complicaciones ${ }^{11}$.

Dos de nuestros pacientes presentaron como resultado en la biopsia un micetoma por aspergilus. Es interesante conocer que la esporas del aspergillum pueden ser introducidas en el antrum del seno maxilar a través de una comunicación oroantral 0 a través de una perforación del canal de una raíz que está en estrecha relación con el seno. Como complicación de los tratamientos de endodoncia se ha sugerido que el llenado excesivo del conducto radicular en tratamiento de conducto puede provocar sobre extensión del material de endodoncia en el seno maxilar, convirtiéndose en el principal factor etiológico de la aspergilosis del seno maxilar en pacientes sanos $^{23}$. Durante la obturación, materiales sólidos o selladores pueden caer al seno maxilar provocando irritación mecánica y reacción inflamatoria. Se ha estudiado que la presencia de óxido de zinc y la pasta de paraformaldehído utilizada en tratamientos radiculares favorece el crecimiento de Aspergillus fumigatus y que la introducción de corticoesteroides en pastas endodónticas puede dar lugar a inmunosupresión local que facilita el crecimiento del hongo ${ }^{24}$.

\section{CONCLUSIONES}

La RSMD es una patología frecuente cuya incidencia se encuentra cercana al $30 \%{ }^{25}$ de los casos de rinosinusitis maxilar. Su presentación clínica es similar a cuadros rinosinusales de otras etiologías lo que puede llevar al diagnóstico errado y muchas veces tardío. La patología dental puede pasar inadvertida tanto en las radiografías dentales como en la tomografía computarizada, por lo que tanto otorrinolaringólogos como odontólogos y radiólogos deben tener presente las limitaciones de los métodos diagnósticos disponibles y buscar dirigidamente patologías dentales que expliquen el cuadro clínico, especialmente en pacientes con historia de rinosinusitis crónica, refractaria a tratamiento habitual y con síntomas máxilo-etmoidales unilaterales. Las bacterias responsables corresponden a 
flora mixta aerobia y anaerobia. El manejo debe ser multidisciplinario tanto por odontólogo como por otorrinolaringólogo de manera de asegurar la resolución del cuadro y prevenir posibles recurrencias y complicaciones. Una combinación de tratamiento médico y quirúrgico es generalmente necesario.

\section{BIBLIOGRAFÍA}

1. Maloney PL, Doku HC. Maxillary sinusitis of odontogenic origin. J Can Dent Assoc (Tor) 1968; 34: 591-603.

2. Albu S, Baciut M. Failures in endoscopic surgery of the maxillary sinus. Otolaryngol Head Neck Surg 2010; 142: 196-201.

3. Melen I, Lindahl L, Andreasson L, Rundcrantz H. Chronic maxillary sinusitis. Definition, diagnosis and relation to dental infections and nasal polyposis. Acta Otolaryngol 1986; 101: 320-7.

4. Hoskinson E, Daniel M, Rowson JE, Jones NS. Evidence of an increase in the incidence of odontogenic sinusitis over the last decade in the UK. J Laryngol Otol 2012; 126(1): 436.

5. Eberhardt Ja, Torabinejad M, Christiansen El. A computed tomographic study of the distances between the maxillary sinus floor and the apices of the maxillary sinus floor and the apices of the maxillary posterior teeth. Oral Surg Oral Med Oral Pathol 1992; 73: 345-6.

6. Menra P, Murad H. Maxillary sinus disease of odontogenic origin. Otolaryngol Clin North Am 2004; 37(2): 347-64.

7. ERICSON S. Conventional and computerized imaging of maxillary sinus pathology related to dental problems. Oral Maxillofac Surg Clin North Am 1992; 4: 153-81.

8. Kretzschmar DP, Kretzschmar JL. Rhinosinusitis: review from a dental perspective. Oral Surg Oral Med Oral Pathol Oral Radiol Endod 2003; 96: 128-35.

9. Arias 0, Barona C, Santos-Marino J, Martínez N, Martínez JM. Meta-analisis of the etiology of odontogenic maxillary sinusitis. Med Oral Patol Oral Cir Bucal 2010; 15 (1): e70-73.

10. Longhini $A B$, Ferguson BJ. Clinical aspects of odontogenic maxillary sinusitis: a case series. Int Forum Allergy Rhinol 2011; 1(5): 409-15.

11. Brook I. Sinusitis of odontogenic origin. Otolaryngol Head Neck Surg 2006; 135(3): 349-5.

12. Lee KC, Lee SJ. Clinical features and treatments of odontogenic sinusitis. Yonsei Med J 2010; 51(6): 932-7.

13. Douglass CW, Valachovic RW, WiJesinha $A$, Chauncey HH, Kapur KK, McNeil BJ. Clinical efficacy of dental radiography in the detection of dental caries and periodontal diseases. Oral Surg Oral Med Oral Pathol 1986; 62: 330-9.

14. Bomel S, Branstetter B, Ferguson B. Frequency of a dental source for acute maxillary sinusitis. Laryngoscope 2009; 119: 580-4.

15. Cymerman JJ, Cymerman DH, O’Dwyer RS. Evaluation of odontogenic maxillary sinusitis using cone-beam computed tomography: three case reports. J Endod 2011; 37: 1465-9.

16. Maillet M, Bowles WR, McClanahan SL, ET AL. Cone-beam computed tomography evaluation of maxillary sinusitis. J Endod 2011; 37: 753-7.

17. NaIR UP, NaIR MK. Maxillary sinusitis of odontogenic origin: cone-beam volumetric computed tomography-aided diagnosis. Oral Surg Oral Med Oral Pathol 2010; 110: e53-e57.

18. Regev E, Smith RA, Perrott DH, Pogrel MA. Maxillary sinus complications related to endosseous implants. Int J Oral Maxillo-Fac Implants 1995; 10: 451-61.

19. Ueda M, KanedA T. Maxillary sinusitis caused by dental implants: report of two cases. J Oral Maxillofac Surg 1992; 50: 285-7.

20. Quiney Re, Brimble M, Hodge M. Maxillary sinusitis from dental osseointegrated implants. J Laryngol Otol 1990; 104: 333-4.

21. BRook I. Microbiology of acute and chronic maxillary sinusitis associated with an odontogenic origin. Laryngoscope 2005; 115(5): 823-5.

22. Puglisi S, Privitera S, Maiolino L, et al. Bacteriological findings and antimicrobial resistance in odontogenic and nonodontogenic 
chronic maxillary sinusitis. J Med Microbiol 2011; 60: 1353-9.

23. Giardino L, Pontieri F, Savoldi E, Tallarigo F. Aspergillusmycetoma of the maxillary sinus secondary to overfilling a root canal. $J$ Endod 2006; 32: 692-4

24. Khongkhunthian P,Reichart PA. Aspergillosis of the maxillary sinus as a complication of overfilling root canal material into the sinus: report of two cases. J Endod 2001; 27: 476-8.

25. Patel NA, Ferguson BJ. Odontogenic sinusitis: an ancient but under-appreciated cause of maxillary sinusitis. Curr Opin Otolaryngol Head Neck Surg 2012; 20(1): 24-8.

Dirección: Claudia Heider C.

Felipe II 4277 Depto. 403. Las Condes, Santiago, Chile.

Teléfono: 5626108667

E mail: claudiaheider@gmail.com 